\title{
Constrained Entropy-based Temperature Control of Waste Heat Systems
}

\author{
Jianhua Zhang, Mifeng Ren and Hong Yue
}

\begin{abstract}
A minimum error entropy controller is developed for superheated vapour temperature control of a waste heat recovery process using Organic Rankine cycles (ORC). A nonlinear dynamic model is briefed for the ORC evaporator to capture the key dynamic characteristics of the process. Considering non-Gaussian disturbance terms, the control objective is proposed to minimize the combined entropy function and the mean value of the squared tracking errors. The controller is designed by taking into account of bounded constraints on input actions. The improved performances of the proposed method in reducing control variation and decreasing tracking error uncertainty are discussed by a comparison with standard PID control through simulation study conducted on an ORC waste heat recovery process.
\end{abstract}

\section{INTRODUCTION}

$\mathrm{O}$ ganic Rankine cycle (ORC) offer certain advantages in thermal processes such as less heat required during the evaporation process, smaller temperature difference between evaporation and condensation processes, evaporation process occurs at lower pressure and lower temperature, expansion process ends in the vapour region, to name a few. In addition, high reliability and flexibility in ORC operations are also attractive features. Therefore, ORC has been widely used to transform low grade energy into electric power from various thermal processes, for example, solar energy, biomass products, geothermal energy, and in particular waste heat recovery [1] Improving performance of ORC systems has been an active research field in the past decades [2]. Research development includes selection of organic working fluid $[3,4]$, modelling of key components in ORC systems [5, 6], performance analysis and optimization of ORC systems [7-9], and experimental study of ORC systems [10].

When a waste heat recovery process operates in ORC, its energy efficiency is closely related to the thermodynamic states of working fluid at various components in the cycle, which can be characterized by operating parameters typically evaporating temperature (or evaporating pressure), condensing temperature, superheated vapour temperature at outlet of the evaporator, and sub-cooling at outlet of the condenser. A proper regulation of these operating parameters in an ORC process is economically essential to improve lifetime and operating efficiency.

Jianhua Zhang is with State Key Laboratory of Alternate Electrical Power System with Renewable Energy Sources, North China Electric Power University, Beijing, 102206, China (email: zjh@ncepu.edu.cn).

Mifeng Ren is with College of Information Engineering, Taiyuan University of Technology, Taiyuan, 030024, China (email: renmifeng@126.com).

Hong Yue is with Department of Electronic and Electrical Engineering, University of Strathclyde, Glasgow G1 1XW, UK (email: hong.yue@strath.ac.uk).

This work was supported by National Basic Research Program of China under Grant (973 Program 2011 CB710706) and China National Science Foundation under Grant (61374052).
However, it is technically challenging to control ORC operating parameters due to complex natures characterized by nonlinearity, uncertainty and disturbances from load and waste heat resources. In [6], two single-loop PID control systems were applied to control an ORC based waste heat recovery process. The evaporating temperature was controlled by manipulating expander speed, while the superheating was controlled by manipulating pump flow rate. Multivariable control strategies were presented for ORC based waste heat utilization processes by incorporating a linear quadratic regulator with a PI controller in [11], and using a generalized predictive controller in [12], respectively. Considering constraints on control input, a constrained model predictive controller is proposed in [13] to control waste heat energy conversion systems (WHECSs).

Superheated vapour temperature is one of the most important parameters in ORC processes. For safety and economic reasons, the temperature of working fluid at the outlet of evaporator must be controlled below a set-up level during the ORC operation process. One challenging issue in practical ORC control is to consider stochastic disturbances rather than deterministic fluctuations in the inlet mass flow rate and also in the temperature of waste heat of the evaporator. In our earlier work [14], under Gaussian disturbance assumptions, an online self-tuning generalized minimum variance (GMV) controller was proposed for a $100 \mathrm{KW}$ waste heat recovery system with ORC.

In this work, we aim to develop controller for this system by considering non-Gaussian disturbance signals. Following recent developments on minimum entropy control of output probability density function (PDF) tracking errors $[10,11,15,16]$, the superheated vapour temperature control system is investigated under the framework of stochastic distribution control, where the constraints on control input is also considered.

The rest of this paper is organized as follows. A moderately complex dynamic model for ORC evaporator is established in Section 2. In Section 3, the proposed controller is developed using the improved minimum error entropy (MEE) criterion and the stability condition of the control system is discussed. Simulation studies of the proposed method in superheated temperature control of ORC process are conducted in Section 4 by comparing with a PID controller. Conclusions are given in Section 5

\section{MODELLING OF ORC EVAPORATOR}

\section{A. ORC Based Waste Heat Recovery Process}

An ORC heat recovery power plant converts waste heat into electrical power through several thermodynamic processes as shown briefly in Fig.1. In this thermodynamic cycle, the organic working fluid R245fa is compressed by a pump from the sub-cooled liquid state at condensation 
pressure (' 1 ') into evaporation pressure ('2'), subsequently, heated up into a superheated vapour state ('3'). The vapour with high temperature and high pressure enters the turbine expander and generates power energy following the expansion process down to a state with lower temperature and pressure (' 4 '). This working fluid is further cooled to liquid state (' 1 ') through the air-cooled condenser.

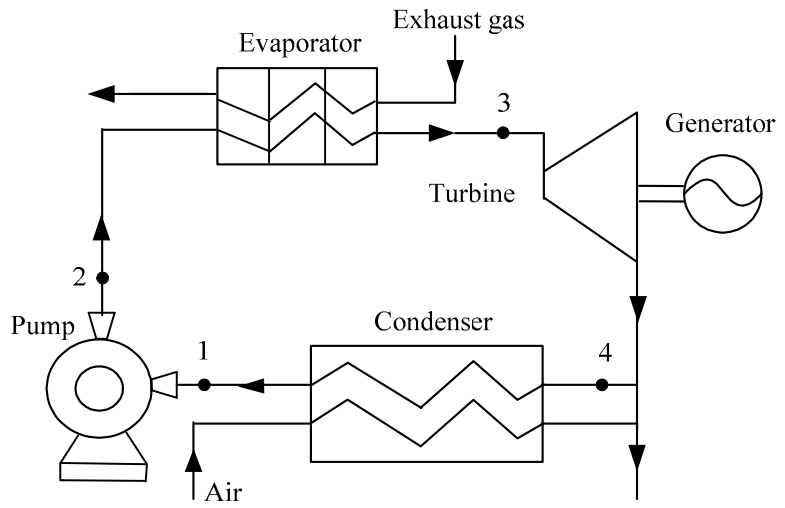

Fig. 1. Illustration of ORC based waste heat recovery

In this work, the model of ORC evaporator is developed from models in our previous work $[11,13]$ by incorporating the random disturbances coming from the inlet mass flow rate and the temperature of exhaust gas. The configuration of the evaporator is considered to be of the cross-flow type with the exhaust gas as the secondary fluid. The evaporator can usually be divided into three zones: a sub-cooled liquid section, a saturated mixture section and a superheated vapour section (see Fig. 2).

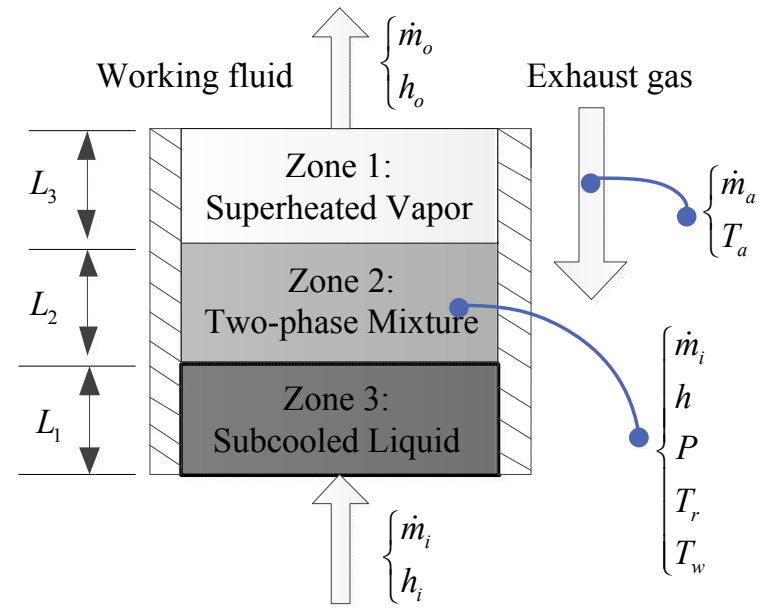

Fig.2. Schematic diagram of evaporator modelling

\section{B. The Side of Working Fluid (Primary Fluid)}

For simplicity, the following assumptions are made for establishing the moving boundary model: (i) the evaporator is a long, thin, horizontal tube; (ii) the working fluid flowing through the evaporator tube can be modelled as a one-dimensional fluid flow; (iii) the pressure drop along the evaporator tube is negligible; (iv) the axial heat conduction of the working fluid is negligible. With these assumptions, the governing partial differential equations (PDEs) for mass and energy conversation of the working fluid can be established as follows. See the nomenclature in appendix for the use of notations, subscripts in modelling.

Mass balance:

$$
\frac{\partial A \rho}{\partial t}+\frac{\partial \dot{m}}{\partial z}=0
$$

Energy balance:

$$
\frac{\partial(\rho A h-A P)}{\partial t}+\frac{\partial \dot{m} h}{\partial z}=\pi D_{i} \alpha_{\mathrm{i}}\left(T_{w}-T_{r}\right)
$$

Differential energy balance at the wall:

$$
\left(C_{p} \rho A\right)_{w} \frac{\partial T_{w}}{\partial t}=\pi D_{i} \alpha_{i}\left(T_{r}-T_{w}\right)+\pi D_{o} \alpha_{o}\left(T_{a}-T_{w}\right)
$$

The boundary conditions of $\dot{m}_{i}, h_{i}$ and $\dot{m}_{o}$ are considered in the governing PDEs of the evaporator dynamics. Equations (1) - (3) are integrated along the axial coordinate within each of the definition regions to build the moving boundary model.

Zone 1. The working fluid in Zone 1 can be considered as incompressible liquid. Hence the mass balance equation is

$$
\dot{m}_{i}=\dot{m}_{\text {int } 1}
$$

By integrating (2) from $z=0$ to $z=L_{1}(t)$, the energy balance equation for Zone 1 can be obtained as follows

$$
\begin{aligned}
& A L_{1}\left[\left(\frac{\rho_{l}}{2} \frac{\mathrm{d} h_{l}}{\mathrm{~d} P}-1\right) \frac{\mathrm{d} P}{\mathrm{~d} t}\right]+\frac{h_{i}-h_{l}}{2} A \rho_{l} \frac{\mathrm{d} L_{1}}{\mathrm{~d} t} \\
= & \alpha_{i 1} \pi D_{i} L_{1}\left(T_{w 1}-T_{r 1}\right)+\dot{m}_{i}\left(h_{i}-h_{l}\right)
\end{aligned}
$$

The energy equation for the tube wall of Zone 1 is

$$
\begin{aligned}
& \left(C_{p} \rho A\right)_{w}\left[\frac{d T_{w 1}}{d t}+\frac{T_{w 1}-T_{w 2}}{L_{1}} \frac{d L_{1}}{d t}\right] \\
= & \alpha_{i 1} \pi D_{i}\left(T_{r 1}-T_{w 1}\right)+\alpha_{o} \pi D_{o}\left(T_{a 1}-T_{w 1}\right)
\end{aligned}
$$

Zone 2. By integrating (1) from $z=L_{1}(t)$ to $z=L_{2}(t)$, the mass balance equation for Zone 2 can be expressed as

$$
\begin{aligned}
& A L_{2} \frac{\mathrm{d} \rho_{2}}{\mathrm{~d} P} \frac{\mathrm{d} P}{\mathrm{~d} t}+A\left(\rho_{2}-\rho_{g}\right) \frac{\mathrm{d} L_{2}}{\mathrm{~d} t}+A\left(\rho_{l}-\rho_{g}\right) \frac{\mathrm{d} L_{1}}{\mathrm{~d} t} \\
= & \dot{m}_{\text {int } 1}-\dot{m}_{\text {int } 2}
\end{aligned}
$$

where $\rho_{2}=\rho_{l}(1-\bar{\gamma})+\rho_{g} \bar{\gamma}$ is the average density of the working fluid in Zone 2. $\bar{\gamma}$ is the mean value of void fraction.

The energy balance equation for Zone 2 is written as

$$
\begin{aligned}
& A L_{2}\left[(1-\bar{\gamma}) \frac{\mathrm{d} \rho_{l} h_{l}}{\mathrm{~d} P}+\bar{\gamma} \frac{\mathrm{d} \rho_{g} h_{g}}{\mathrm{~d} P}-1\right] \frac{\mathrm{d} P}{\mathrm{~d} t} \\
& +A(1-\bar{\gamma})\left(\rho_{l} h_{l}-\rho_{g} h_{g}\right) \frac{\mathrm{d} L_{2}}{\mathrm{~d} t}+A\left(\rho_{l} h_{l}-\rho_{g} h_{g}\right) \frac{\mathrm{d} L_{1}}{\mathrm{~d} t} \\
= & \dot{m}_{\text {int } 1} h_{l}-\dot{m}_{\text {int } 2} h_{g}+\pi D_{i} \alpha_{i 2} L_{2}\left(T_{w 2}-T_{r 2}\right)
\end{aligned}
$$

The energy equation for the tube wall of Zone 2 is

$$
\left(C_{p} \rho A\right)_{w} \frac{\mathrm{d} T_{w 2}}{\mathrm{~d} t}=\alpha_{i 2} \pi D_{i}\left(T_{r 2}-T_{w 2}\right)+\alpha_{o} \pi D_{o}\left(T_{a 2}-T_{w 2}\right)
$$

Zone 3. The mass balance equation can be obtained by integrating (1) from $z=L_{1}(t)+L_{2}(t)$ to $z=L$, which gives 
$A L_{3} \frac{\mathrm{d} \rho_{3}}{\mathrm{~d} P} \frac{\mathrm{d} P}{\mathrm{~d} t}+A\left(\rho_{g}-\rho_{3}\right)\left(\frac{\mathrm{d} L_{2}}{\mathrm{~d} t}+\frac{\mathrm{d} L_{1}}{\mathrm{~d} t}\right)=\dot{m}_{\mathrm{int} 2}-\dot{m}_{o}$

The energy balance equation for Zone 3 can be formulated by

$$
\begin{aligned}
& A L_{3}\left[\left(\frac{\rho_{3}}{2} \frac{\mathrm{d} h_{g}}{\mathrm{~d} P}-1\right) \frac{\mathrm{d} P}{\mathrm{~d} t}+\frac{\rho_{3}}{2} \frac{\mathrm{d} h_{o}}{\mathrm{~d} t}\right]=\alpha_{i 3} \pi D_{i} L_{3}\left(T_{w 3}-T_{r 2}\right) \\
& -\frac{1}{2}\left(\dot{m}_{o}+\dot{m}_{\mathrm{int} 2}-A \rho_{g}\left(\frac{\mathrm{d} L_{2}}{\mathrm{~d} t}+\frac{\mathrm{d} L_{1}}{\mathrm{~d} t}\right)\right)\left(h_{o}-h_{g}\right)
\end{aligned}
$$

The energy equation for the tube wall of Zone 3 is

$$
\begin{aligned}
& \left(C_{p} \rho A\right)_{w}\left[\frac{\mathrm{d} T_{w 2}}{\mathrm{~d} t}+\frac{T_{w 2}-T_{w 3}}{L_{3}}\left(\frac{\mathrm{d} L_{2}}{\mathrm{~d} t}+\frac{\mathrm{d} L_{1}}{\mathrm{~d} t}\right)\right] \\
= & \alpha_{i 3} \pi D_{i}\left(T_{r 3}-T_{w 3}\right)+\alpha_{o} \pi D_{o}\left(T_{a 3}-T_{w 3}\right)
\end{aligned}
$$

\section{The Side of Exhaust Gas (Secondary Fluid)}

Since the mass flow rate and the pressure on the side of exhaust gas can be regarded as invariant, only the energy conservation of exhaust gas is considered. The PDE that governs the energy balance of the exhaust gas flowing through the evaporator can be described by:

$$
\frac{\partial(\rho A h-A P)}{\partial t}+\frac{\partial \dot{m} h}{\partial z}=\pi D_{o} \alpha_{o}\left(T_{w}-T_{r}\right)
$$

Similar to the modelling of the working fluid, the energy balance equations for exhaust gas in the three zones can then be integrated as follows:

$$
\begin{aligned}
& A_{a} L_{1} \rho_{a} C_{p} \frac{\mathrm{d} T_{a 1}}{\mathrm{~d} t}+A_{a} \rho_{a}\left(\frac{h_{c}-h_{a, \text { int } 1}}{2}\right) \frac{\mathrm{d} L_{1}}{\mathrm{~d} t} \\
= & \dot{m}_{a} h_{a, \text { int } 1}-\dot{m}_{a} h_{c}+\pi D_{o} \alpha_{o} L_{1}\left(T_{w 1}-T_{a 1}\right) \\
& A_{a} L_{2} \rho_{a} C_{p} \frac{\mathrm{d} T_{a 2}}{\mathrm{~d} t}+A_{a} \rho_{a}\left(h_{a, \text { int } 1}-h_{a, \text { int } 2}\right)\left(\frac{\mathrm{d} L_{1}}{\mathrm{~d} t}+0.5 \frac{\mathrm{d} L_{2}}{\mathrm{~d} t}\right) \\
= & \dot{m}_{a} h_{a, \text { int } 2}-\dot{m}_{a} h_{a, \text { int } 1}+\pi D_{o} \alpha_{o} L_{2}\left(T_{w 2}-T_{a 2}\right) \\
& A_{a} L_{3} \rho_{a} C_{p} \frac{d T_{a 3}}{d t}-A_{a} \rho_{a} \frac{h_{a, i}-h_{a, \text { int } 2}}{2}\left(\frac{\mathrm{d} L_{1}}{\mathrm{~d} t}+\frac{\mathrm{d} L_{2}}{\mathrm{~d} t}\right) \\
= & \dot{m}_{a} h_{a, i}-\dot{m}_{a} h_{a, \text { int } 2}+\pi D_{o} \alpha_{o} L_{3}\left(T_{w 3}-T_{a 3}\right)
\end{aligned}
$$

By defining state variables and control inputs, a nonlinear state-space model can be established from the above first-principle model. In this system, the quality of the exhaust gas has influence on the outlet temperature of the working fluid from the evaporator, $T_{s h}$. The factors influencing the quality of exhaust gas include the temperature at the inlet of evaporator, the mass flow rate and the chemical composition of exhaust gas in ORC processes. It should be noted that the disturbances induced by the inlet temperature and the mass flow rate of exhaust gas are not necessarily Gaussian.

The discretized model of the ORC process can be written in a general form of the following non-linear ARMAX equation:

$$
y_{k}=F\left(y_{k-1}, \cdots, y_{k-n}, u_{k}, u_{k-1}, \cdots, u_{k-m}, v_{k}\right)
$$

where $F(\cdot)$ is a nonlinear function representing system dynamics, $k$ is the time index, $y_{k}$ is the superheated vapor temperature $\left(T_{s h}\right)$ at time $k ; u_{k}$ is the speed of the compression pump ( $\omega) ; v_{k}=\left[\dot{m}_{a}(k) T_{a}(k)\right]^{\mathrm{T}}$ is the external bounded disturbance vector with known PDF $\gamma_{v}(\cdot)$. The mass flow rate $\dot{m}_{a}(k)$ and the temperature of exhaust gas at the inlet of the evaporator, $T_{a}(k)$, are assumed to be independent of each other. Since the two disturbance terms are considered to be non-Gaussian, the output $y_{k}$ is also a non-Gaussian stochastic variable. The discrete-time input-output model in (17) can be established from data produced by the nonlinear first-principle model using parameter estimation techniques.

\section{MINIMUM ENTROPY CONTROLLER}

\section{A. Schematic Diagram and Performance Index}

In an ORC heat recovery process, the output power of the expander increases with the increase of the superheated vapour temperature, but the superheated vapour temperature is not allowed to exceed the critical temperature level. A control system is proposed as shown in Fig. 3.

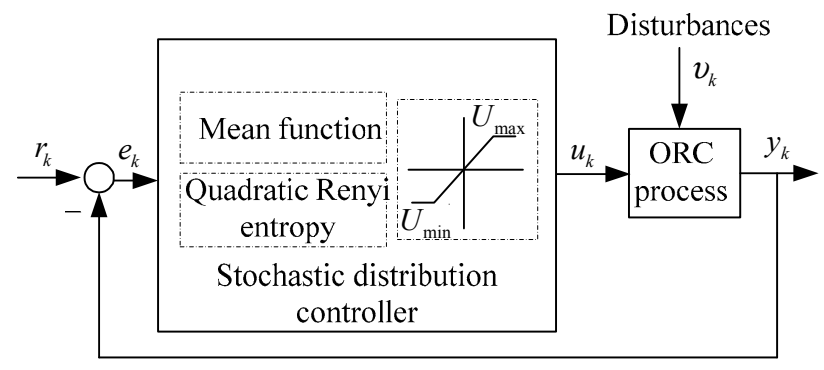

Fig. 3. Schematic diagram of the proposed control system

At time $k$, the tracking error of the closed-loop system is defined as $e_{k}=r_{k}-y_{k}$, thus

$$
\begin{aligned}
e_{k} & =y_{k}-r_{k}=F\left(y_{k-1}, \cdots, y_{k-n}, u_{k}, u_{k-1}, \cdots, u_{k-m}, v_{k}\right)-r_{k} \\
& =g\left(y_{k-1}, \cdots, y_{k-n}, u_{k}, u_{k-1}, \cdots, u_{k-m}, v_{k}, r_{k}\right) \\
& =g\left(\eta_{k}, u_{k}, v_{k}\right)
\end{aligned}
$$

where $g(\cdot)$ is a known nonlinear function, $\eta_{k}=\left(y_{k-1}, \cdots, y_{k-n}, u_{k-1}, \cdots, u_{k-m}, r_{k}\right)^{\mathrm{T}}$ is a known term at time $k$. Then, the PDF of the tracking error can be formulated as

$$
\gamma_{e_{k}}\left(\eta_{k}, u_{k}, x\right)=\gamma_{v}\left(g^{-1}\left(\eta_{k}, u_{k}, x\right)\right)\left|\frac{\mathrm{d} g^{-1}\left(\eta_{k}, u_{k}, x\right)}{\mathrm{d} x}\right|
$$

The entropy of the tracking error can be used to represent the degree of randomness in the stochastic signal [15]. For simplicity, the quadratic information potential $V\left(e_{k}\right)=\int \gamma_{e_{k}}^{2}\left(\eta_{k}, u_{k}, x\right) \mathrm{d} x$ is an alternative way to characterize the quadratic entropy of the tracking error (entropy is a monotonic decreasing function of the quadratic information potential). The mean value of the squared tracking error is $\mathrm{E}\left\{e_{k}^{2}\right\}=\int\left(\tau^{2} \gamma_{e_{k}}\left(\eta_{k}, u_{k}, x\right)\right) \mathrm{d} x$ and it should also be included to minimize the level of the tracking error. 
The following performance index is proposed for controller design:

$$
J\left(u_{k}\right)=-R_{1} V\left(e_{k}\right)+R_{2} \mathrm{E}\left\{e_{k}^{2}\right\}+\frac{1}{2} R_{3} u_{k}^{2}
$$

where $R_{1}$ and $R_{2}$ are weights assigned for the information potential, the mean value of the tracking error function, respectively, and $R_{3}$ is the weighting factor for the control input. The mean value of the squared tracking error can be estimated from the output measurement. The information potential of the tracking error, $V\left(e_{k}\right)$, can also be estimated using the error series data within a sliding window [16].

\section{B. Constrained Optimal Controller Design}

To address the physical bounds on the rotating speed of the compressing pump in practical ORC processes, the following constraint is imposed on the control input

$$
U_{\min } \leq u_{k} \leq U_{\max }
$$

where $U_{\min }$ and $U_{\max }$ are lower and upper bounds for $u_{k}$. The following constrained optimization problem is thus formulated:

$$
\left\{\begin{aligned}
& \min J\left(u_{k}\right)= \int\left(-R_{1} \gamma_{e_{k}}^{2}\left(\eta_{k}, u_{k}, x\right)+R_{2} \tau^{2} \gamma_{e_{k}}\left(\eta_{k}, u_{k}, x\right)\right) \mathrm{d} x \\
&+\frac{1}{2} R_{3} u_{k}^{2} \\
& \text { s.t. } U_{\max }-u_{k} \geq 0, \quad u_{k}-U_{\min } \geq 0
\end{aligned}\right.
$$

A penalty function method is employed to solve this constrained nonlinear programming problem.

$$
\begin{aligned}
J_{\text {new }}\left(u_{k}, M_{k}\right) & =J\left(u_{k}\right)+M_{k} \cdot\left[\min \left(0, U_{\max }-u_{k}\right)\right]^{2} \\
& +M_{k} \cdot\left[\min \left(0, u_{k}-U_{\min }\right)\right]^{2}
\end{aligned}
$$

where the penalty factor $M_{k}$ is a positive number. A recursive decreasing gradient method is used to find the optimal solution in the following steps.

Step 1: Denote $k:=1$, choose the initial control input $u_{0}$ and the penalty factor $M_{1}$, set the magnification factor $\beta>1$ and the accuracy level $\varepsilon>0$.

Step 2: Take $u_{k-1}$ as the initial point and calculate the following gradient for $u_{k}, \nabla J_{\text {new }}\left(u_{k}\right)=\partial J_{\text {new }}\left(u_{k}\right) / \partial u_{k}$. If $\nabla J_{\text {new }}\left(u_{k}\right)<\varepsilon$, terminate the optimization process and take the optimal solution $u_{k}^{*}=u_{k}$, otherwise go to Step 3 .

Step 3: Set the step length $\lambda_{k}$, the next control input is

$$
u_{k+1}=u_{k}-\lambda_{k} \nabla J_{\text {new }}\left(u_{k}\right)
$$

Step 4: Set $k:=k+1$, turn to Step 2. Should a solution exist, eventually, the optimal solution $u_{k}^{*}$ can be obtained to minimize $J_{\text {new }}$ in (23).
Step 5: If

$M_{k} \cdot\left(\left[\min \left(0, U_{\max }-u_{k}\right)\right]^{2}+\left[\min \left(0, u_{k}-U_{\min }\right)\right]^{2}\right)<\varepsilon$, terminate the optimization process and obtain the optimal solution $u_{k}^{*}=u_{k}$. Otherwise, set $M_{k+1}=\beta M_{k}, k=k+1$ and turn to Step 2.

\section{Stability Analysis}

The stability analysis of the closed loop system is discussed using the linearized model. The nonlinear inputoutput model in (17) is linearized to read

$$
\Delta y_{k}=\sum_{i=1}^{n} \frac{\partial F}{\partial y_{k-i}} \Delta y_{k-i}+\sum_{j=0}^{m} \frac{\partial F}{\partial u_{k-j}} \Delta u_{k-j}+\frac{\partial F}{\partial v_{1 k}} \Delta v_{1 k}+\frac{\partial F}{\partial v_{2 k}} \Delta v_{2 k}
$$

where $\quad \Delta y_{k}=y_{k}-y_{k-1} \quad, \quad \Delta u_{k}=u_{k}-u_{k-1} \quad$ and $\Delta v_{i k}=v_{i k}-v_{i k-1}(i=1,2)$. Applying the unit backward shift operation $z^{-1}$ to both sides of (25),

$$
\begin{gathered}
\\
\qquad\left(1-\sum_{i=1}^{n} \frac{\partial F}{\partial y_{k-i}} z^{-i}\right) \Delta y_{k} \\
=\left(\sum_{j=0}^{m} \frac{\partial F}{\partial u_{k-j}} z^{-j}\right) \Delta u_{k}+\frac{\partial F}{\partial v_{1 k}} \Delta v_{1 k}+\frac{\partial F}{\partial v_{2 k}} \Delta v_{2 k} \\
\text { Denote } N\left(z^{-1}, k\right)=1-\sum_{i=1}^{n} \frac{\partial F}{\partial y_{k-i}} z^{-i} \\
\xi_{k}=\left(\sum_{j=0}^{m} \frac{\partial F}{\partial u_{k-j}} z^{-j}\right) \Delta u_{k}+\frac{\partial F}{\partial v_{1 k}} \Delta v_{1 k}+\frac{\partial F}{\partial v_{2 k}} \Delta v_{2 k}, \text { then } \\
N\left(z^{-1}, k\right) \Delta y_{k}=\xi_{k}
\end{gathered}
$$

and

It can be seen from (21) that $\left|\Delta u_{k}\right| \leq U_{\max }-U_{\text {min }}$. Moreover, $\Delta v_{1 k}$ and $\Delta v_{2 k}$ are bounded terms. Therefore, $\xi_{k}$ is also bounded. Let $N\left(z^{-1}, k\right)=1-\sum_{i=1}^{n} \alpha_{i}(k) z^{-i}$ and $\mathbf{X}(k)=\left[\Delta y_{k-n} \Delta y_{k-n+1} \cdots \Delta y_{k-1}\right]^{\mathrm{T}}$, the following state-space representation can then be formulated as

$$
\mathbf{X}(k+1)=\mathbf{A}(k) \mathbf{X}(k)+\mathbf{B}(k) \xi_{k}
$$

where $\quad \mathbf{A}(k)=\left[\begin{array}{cccc}0 & 1 & \cdots & 0 \\ \vdots & \vdots & \ddots & \vdots \\ 0 & 0 & \cdots & 1 \\ \alpha_{n}(k) & \alpha_{n-1}(k) & \cdots & \alpha_{1}(k)\end{array}\right]$

$\mathbf{B}(k)=\left[\begin{array}{c}0 \\ \vdots \\ 0 \\ 1\end{array}\right]$. If $\Delta y_{k}$ is bounded, then the linearized closed-

loop system is stable. Consequently, the closed-loop stability condition for the nonlinear and non-Gaussian stochastic system (17) with the constrained input (21) is $\|\mathbf{A}(k)\|<1$. 
The proposed optimal control approach is applied to superheated vapor temperature control of an ORC process. For comparison purpose, an optimal PID controller, $G_{P I D}(s)=k_{p}+k_{i} / s+k_{d} s$, is tuned by Matlab software. The best tuned PID parameters are $k_{p}=3.98 \times 10^{-4}$, $k_{i}=6.64 \times 10^{-4}$ and $k_{d}=0.03$. In this simulation, the sampling period is $T_{s}=1 \mathrm{~s}$. The control inputs of both the proposed controller and the PID controller are limited by a range of $2950 \leq u_{k} \leq 3050 \mathrm{r} / \mathrm{min}$, the initial control input is $u_{0}=3042 \mathrm{r} / \mathrm{min}$. Non-Gaussian disturbances imposed on the mass flow rate and the temperature of exhaust gas at inlet of the evaporator can be assumed to follow $\beta$ distribution with the following PDFs: $\gamma_{v_{1 k}}(x)=$

$\left\{\left[0.02^{a_{1}+b_{1}-1} \beta\left(a_{1}, b_{1}\right)\right]^{-1}(x+0.01)^{a_{1}-1}(0.01-x)^{b_{1}-1}, x \in[-0.01,0.01]\right.$ $\{0$, otherwise

$\gamma_{v_{2 k}}(x)=$

$\int\left[0.2^{a_{2}+b_{2}-1} \beta\left(a_{2}, b_{2}\right)\right]^{-1}(x+0.1)^{a_{2}-1}(0.1-x)^{b_{2}-1}, x \in[-0.1,0.1]$ 0 , otherwise

where $a_{1}=10, b_{1}=4, a_{2}=3, b_{2}=8$. The distributions of the two disturbance terms can be seen in Fig. 4 .
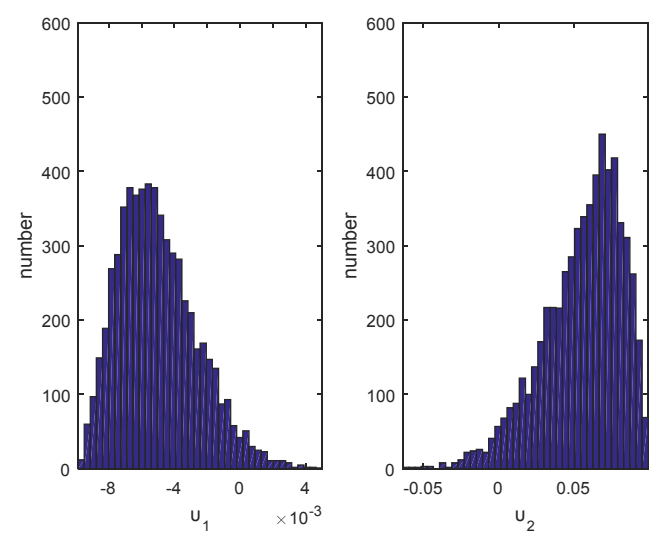

Fig. 4. Distribution of the two disturbance inputs

The ORC process operates at a steady state before $50 \mathrm{~s}$, a step change is introduced to the set point of the superheated vapour temperature at $50 \mathrm{~s}$ from $137.6^{\circ} \mathrm{C}$ to $142.6^{\circ} \mathrm{C}$. The set-point change and the time responses of the superheated vapour temperature under the proposed control and the PID control are shown in Fig. 5. The red dash-dot line shows the set point variation. The blue dotted line and the green solid line represent temperature responses using the PID controller and the proposed control algorithm, respectively. It can be seen that both control methods are able to stabilize the superheated vapour temperature around the set point. The proposed control algorithm obtains smaller overshoot and shorter settling time compared with the PID controller.

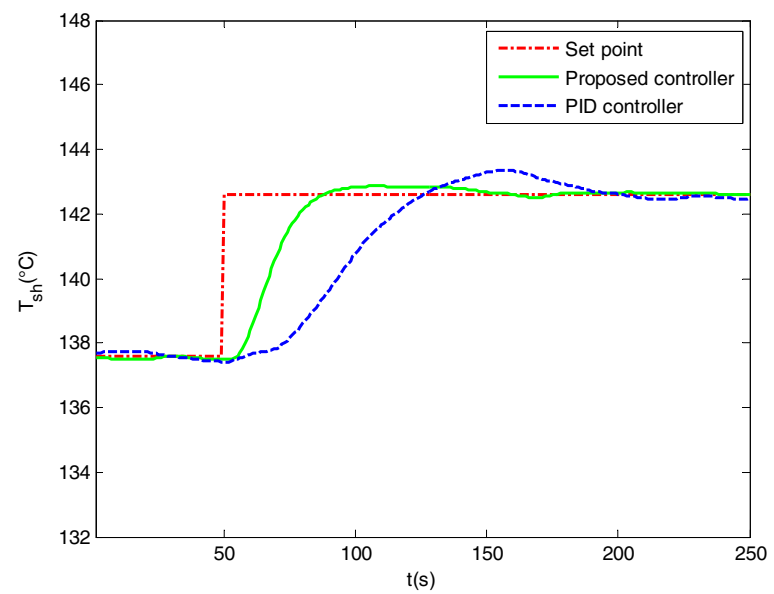

Fig. 5. Response of superheated vapour temperature

It can be observed from Fig. 6 and 7 that the PDFs of the tracking errors become narrower and sharper over the control process using both PID and the proposed control methods, which means both controllers can drive the system towards the direction with a smaller randomness. With a further comparison, it can be seen from Fig. 6 that the PDF of the tracking error approaches to zero under the proposed controller, and the PDF becomes narrow and sharp at $\mathrm{t}=80 \mathrm{~s}$. However, at $\mathrm{t}=80 \mathrm{~s}$, the PDF of the tracking error under PID controller still largely deviates from zero. The constrained control signals are shown in Fig. 8, where the blue dotted line is the control input obtained by the proposed control law, and the green solid line is the control input from the PID controller. With the constraint condition included in the controller design, the control signal from the proposed algorithm stays away from the boundaries during the whole control process. In summary, compared with the PID control, the simulation results demonstrate that the proposed algorithm has achieved shorter settling time, smaller overshoot and less uncertainty in process response.

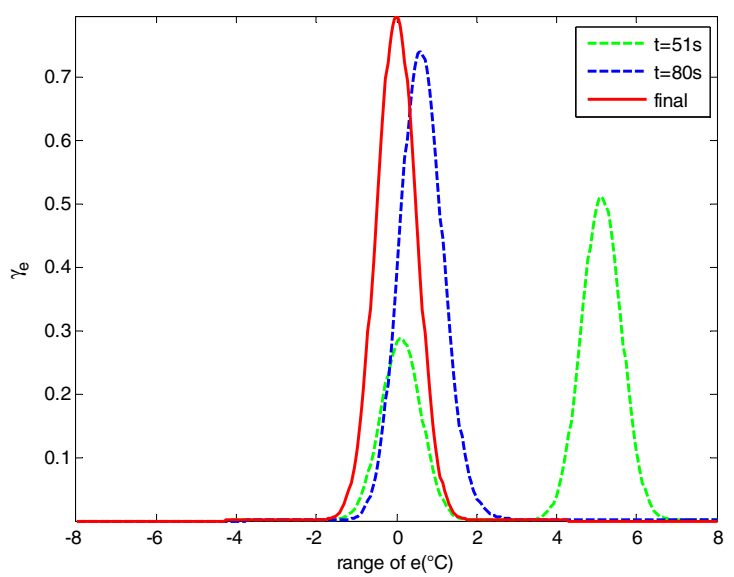

Fig. 6. Selected PDFs under the proposed control 


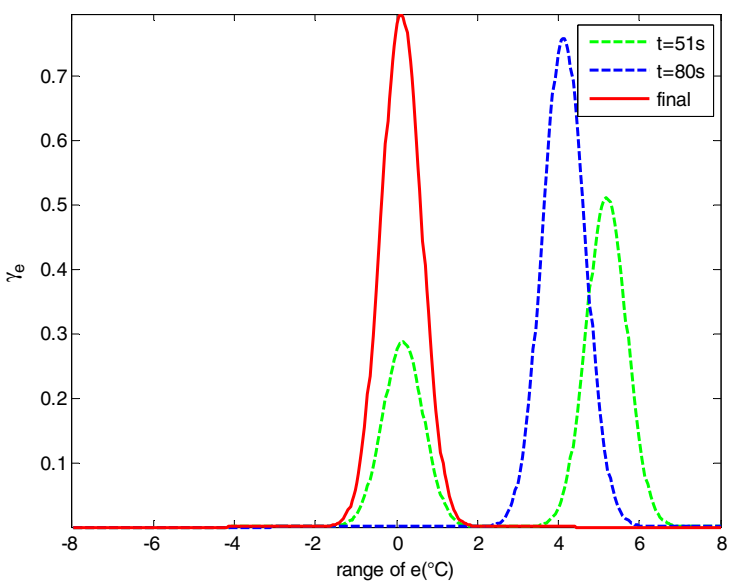

Fig. 7. Selected PDFs under PID control

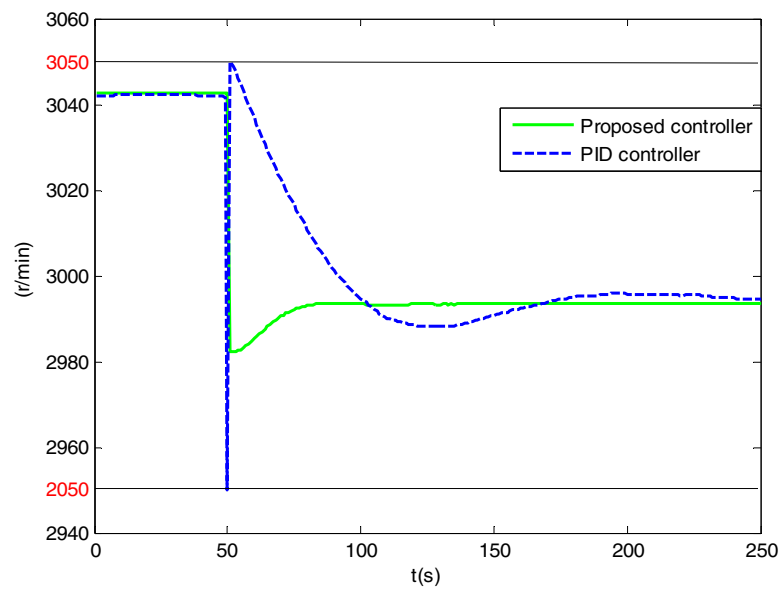

Fig. 8. Time profiles of control input

\section{CONCLUSIONS}

In this paper, a generalized minimum entropy controller is proposed to control the superheated vapour temperature in ORC process. A mathematical model is presented to describe the evaporator dynamics of ORC process, where the disturbances coming from the mass flow rate and the temperature of exhaust gas are considered to be nonGaussian signals. In controller design, the performance index is formed by the entropy or information potential of the output tracking error rather than the mean squared error normally used for Gaussian processes. Accordingly, the superheated vapour temperature control problem is handled in the stochastic control framework by considering bounded constraints on control input. To solve the constrained nonlinear programming optimization problem, the penalty function method is adopted to obtain the recursive optimal control input subject to bounded constraints. When applying the proposed controller and a fine-tuned PID controller to the simulation study of ORC process, both controllers can receive reasonable tracking performance in superheated temperature control, but the proposed controller is capable of handling the constraints on control input, reducing the control variations, and also decreasing dispersion of the tracking error distribution in the superheated vapour temperature with better dynamic response.

\section{REFERENCES}

[1] F. Vélez, J.J. Segovia, M.C. Martín, G. Antolín, F. Chejne, A. Quijano, "A technical, economical and market review of organic Rankine cycles for the conversion of low-grade heat for power generation," Renewable and Sustainable Energy Reviews, vol. 16, no. 6, pp. 4175-4189, Aug. 2012.

[2] B.F. Tchanche, G. Lambrinos, A. Frangoudakis, G. Papadakis, "Low-grade heat conversion into power using organic Rankine cycles - A review of various applications, " Renewable and Sustainable Energy Reviews, vol. 15, no. 8, pp. 3963-3979, Oct. 2011.

[3] A.I. Papadopoulos, M. Stijepovic, P. Linke, "On the systematic design and selection of optimal working fluids for Organic Rankine Cycles," Applied Thermal Engineering, vol. 30, no. 6-7, pp. 760-769, May. 2010.

[4] E.H. Wang, H.G. Zhang, B.Y. Fan, M.G. Ouyang, Y. Zhao, Q.H. Mu, "Study of working fluid selection of organic Rankine cycle (ORC) for engine waste heat recovery," Energy, vol. 36, no. 5, pp. 3406-3418, May. 2011.

[5] J.M. Luján, J.R. Serrano, V. Dolz, J. Sánchez, "Model of the expansion process for R245fa in an Organic Rankine Cycle (ORC),” Applied Thermal Engineering, vol. 40, pp. 248-257, Jul. 2012.

[6] S. Quoilin, R. Aumann, A. Grill, A. Schuster, V. Lemort, H. Spliethoff, " Dynamic modeling and optimal control strategy of waste heat recovery Organic Rankine Cycles," | Applied Energy, vol. 88, no. 6, pp. 2183-2190, Jun. 2011.

[7] S. Quoilin, S. Declaye, B.F. Tchanche, V. Lemort, " Thermo-economic optimization of waste heat recovery Organic Rankine Cycles," Applied Thermal Engineering, vol. 31, no. 14-15, pp. 2885-2893, Oct. 2011.

[8] J.P. Roy, M.K. Mishra, A. Misra, “Performance analysis of an Organic Rankine Cycle with superheating under different heat source temperature conditions," Applied Energy, vol. 88, no. 9, pp. 2995 3004, Sep. 2011.

[9] S.J. Zhang, H.X. Wang, T. Guo, “ Performance comparison and parametric optimization of subcritical Organic Rankine Cycle (ORC) and transcritical power cycle system for low-temperature geothermal power generation," Applied Energy, vol. 88, no. 8, pp. 27402754, Aug. 2011.

[10] S.H. Kang, “Design and experimental study of ORC (organic Rankine cycle) and radial turbine using R245fa working fluid," Energy, vol. 41, no. 1, pp. 514-524, May. 2012.

[11] J. Zhang, W. Zhang, G. Hou, F. Fang, “Dynamic modeling and multivariable control of organic Rankine cycles in waste heat utilizing processes," Computers \& Mathematics with Applications, vol. 64, no. 5, pp. 908-921, Sep. 2012. 
[12] J. Zhang, Y. Zhou, Y. Li, G. Hou, F. Fang, "Generalized predictive control applied in waste heat recovery power plants," Applied Energy, vol. 102, pp. 320-326, Feb. 2013.

[13] J. Zhang, Y. Zhou, R. Wang, J. Xu, F. Fang, "Modeling and constrained multivariable predictive control for ORC (Organic Rankine Cycle) based waste heat energy conversion systems," Energy, vol. 66, pp. 128-138, Mar. 2014.

[14] G. Hou, S. Bi, M. Lin, J. Zhang, J. Xu, “Minimum variance control of organic Rankine cycle based waste heat recovery," Energy Conversion and Management, vol. 86, pp. 576-586, Oct. 2014.

[15] H. Yue, H. Wang, “Minimum entropy control of closed-loop tracking errors for dynamic stochastic systems," IEEE Transactions on Automatic Control, vol. 48, no. 1, pp. 118-122, 2003.

[16] J. Zhang, C.C. Chu, J. Munoz, J. Chen, “Minimum entropy based run-to-run control for semiconductor processes with uncertain metrology delay," Journal of Process Control, vol. 19, no. 10, pp. 1688-1697, Dec. 2009.

\section{APPENDIX}

\section{NOMENCLATURE}

\begin{tabular}{|c|c|}
\hline$A$ & Area $\left[\mathrm{m}^{2}\right]$ \\
\hline$m$ & Mass [kg] \\
\hline$\dot{m}$ & Mass flow rate $[\mathrm{kg} / \mathrm{s}]$ \\
\hline$P$ & Pressure $[\mathrm{kPa}]$ \\
\hline$h$ & Specific enthalpy $[\mathrm{J} / \mathrm{kg}]$ \\
\hline$D$ & Diameter $[\mathrm{m}]$ \\
\hline$\alpha$ & Heat transfer coefficient $\left[\mathrm{W} /\left(\mathrm{m} \cdot{ }^{\circ} \mathrm{C}\right)\right]$ \\
\hline$T$ & Temperature $\left[{ }^{\circ} \mathrm{C}\right]$ \\
\hline $\bar{\gamma}$ & Mean value of void fraction [-] \\
\hline$\rho$ & Density $\left[\mathrm{kg} / \mathrm{m}^{3}\right]$ \\
\hline$L$ & Length of region $[\mathrm{m}]$ \\
\hline$C_{p}$ & Specific heat capacity $[\mathrm{J} / \mathrm{kg}]$ \\
\hline$\omega$ & Pump speed (r/min) \\
\hline$v$ & Disturbance \\
\hline \multicolumn{2}{|c|}{ Subscripts } \\
\hline$a$ & Exhaust gas \\
\hline$w$ & Wall \\
\hline$r$ & Organic working fluid \\
\hline$c$ & Condenser \\
\hline$p$ & Pump \\
\hline$g$ & Saturated vapor \\
\hline$l$ & Saturated liquid \\
\hline$s h$ & Superheated vapor \\
\hline$i$ & Inlet or inner \\
\hline$o$ & Outlet or outer \\
\hline Int 1 & $\begin{array}{l}\text { The interface of liquid region and liquid- } \\
\text { vapor mixture region }\end{array}$ \\
\hline Int 2 & $\begin{array}{l}\text { The interface of liquid-vapor mixture } \\
\text { region and Superheated vapor region }\end{array}$ \\
\hline 1 & Sub-cooled liquid region \\
\hline 2 & Liquid-vapor mixture region \\
\hline 3 & Superheated vapor region \\
\hline
\end{tabular}

